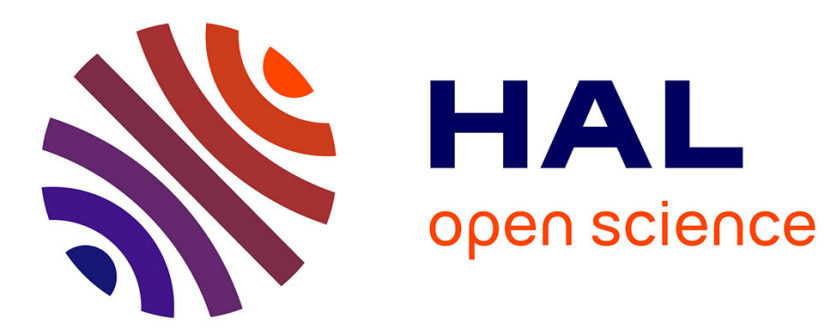

\title{
An electronic equivalent scheme of a Josephson junction
} C.K. Bak

\section{To cite this version:}

C.K. Bak. An electronic equivalent scheme of a Josephson junction. Revue de Physique Appliquée, 1974, 9 (1), pp.15-18. 10.1051/rphysap:019740090101500 . jpa-00243728

\section{HAL Id: jpa-00243728 https://hal.science/jpa-00243728}

Submitted on 1 Jan 1974

HAL is a multi-disciplinary open access archive for the deposit and dissemination of scientific research documents, whether they are published or not. The documents may come from teaching and research institutions in France or abroad, or from public or private research centers.
L'archive ouverte pluridisciplinaire HAL, est destinée au dépôt et à la diffusion de documents scientifiques de niveau recherche, publiés ou non, émanant des établissements d'enseignement et de recherche français ou étrangers, des laboratoires publics ou privés. 


\title{
AN ELECTRONIC EQUIVALENT SCHEME OF A JOSEPHSON JUNCTION
}

\author{
C. K. BAK \\ Physics Laboratory I, The Technical University of Denmark \\ DK-2800 Lyngby, Denmark
}

\begin{abstract}
Résumé. - On montre qu'une boucle de contre-réaction de phase représente assez bien une jonction réelle. De nombreux phénomènes observés dans les jonctions Josephson sont facilement identifiés à l'aide de ce "circuit équivalent ». On montre ensuite que l'adjonction d'une fréquence de coupure haute dans le gain de boucle produit un terme de conductivité semblable au terme en $\cos \varphi$ observé expérimentalement dans des expériences de résonance de plasma.

Abstract. - It is shown that a phase-locked loop is a rather close approximation to a real junction. A lot of phenomena observed in Josephson junctions are easily identified in the « equivalent circuit ». It is further shown that the inclusion of an upper cut-off frequency in the loop gain causes a conductivity term similar to the $\cos \varphi$-term experimentally observed in plasma resonance experiments.
\end{abstract}

1. Introduction. - The basic form of a phaselocked loop is shown in figure 1 . The voltage controlled oscillator (VCO) generates a signal

$$
V_{\mathrm{vco}}=V_{1} \sin \left(\omega_{0} t+k \int V_{\mathrm{i}} \mathrm{d} t\right)
$$

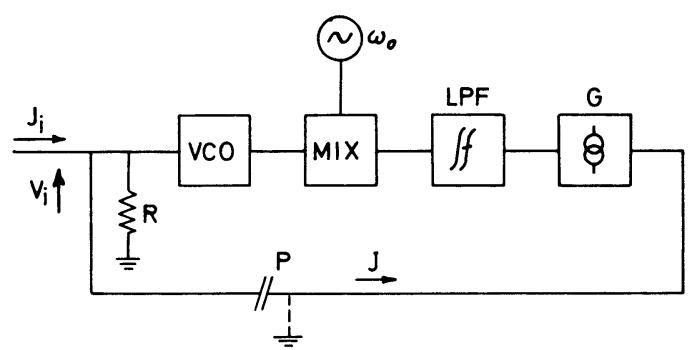

FIG. 1. - Block diagram of a phase-locked loop. VCO $=$ voltage controlled oscillator, MIX $=$ mixer, $\mathrm{LPF}=$ low-pass filter, and $G=$ current generator.

at angular frequency

$$
\omega_{\mathrm{VCO}}=\omega_{0}+k V_{\mathrm{i}},
$$

$k$ being a constant for the VCO and $V_{\mathrm{i}}$ the input voltage. The output of the VCO and a carrier signal $V_{2} \cos \omega_{0} t$ are fed to the two inputs of a balanced mixer. The mixer output is proportional to the product of the two input voltages :

$$
\begin{aligned}
V_{\mathrm{MIX}}= & \alpha V_{1} V_{2} \sin \left(\omega_{0} t+k \int V_{\mathrm{i}} \mathrm{d} t\right) \cos \omega_{0} t \\
= & \frac{\alpha}{2} V_{1} V_{2} \times \\
& \times\left[\sin \left(2 \omega_{0} t+k \int V_{\mathrm{i}} \mathrm{d} t\right)+\sin k \int V_{\mathrm{i}} \mathrm{d} t\right] .
\end{aligned}
$$

The carrier frequency is chosen sufficiently high to ensure $\left|k \cdot V_{\mathrm{i}}\right|_{\max } \ll \omega_{0}$. It is then possible to choose a lowpass filter with cut-off frequency $\omega_{c}$ to select the voltage

$$
\frac{\alpha}{2} V_{1} V_{2} \sin k \int V_{\mathrm{i}} \mathrm{d} t
$$

The output from the current amplifier (transconductance $G$ ) is then

$$
J=J_{1} \sin k \int V_{\mathrm{i}} \mathrm{d} t,
$$

where $J_{1}=(\alpha / 2) G V_{1} V_{2}$.

The total current drawn by the input is

$$
\begin{aligned}
J_{\mathrm{i}} & =J_{1} \sin \varphi+\frac{V_{\mathrm{i}}}{R} \\
\frac{\mathrm{d} \varphi}{\mathrm{d} t} & =k V_{\mathrm{i}} .
\end{aligned}
$$

For simplicity the voltage from the VCO as well as the carrier have been taken to be sinusoidal, producing a $\sin \varphi$ dependence in eq. (1). Other functional dependencies can be realized using appropriate wave forms [1].

Let the VCO output $V_{1}$ be a symmetrical square wave and the carrier a periodic waveform $F\left(\varphi_{0}\right)$, $\varphi_{0}=\omega_{0} t$, with the property $F\left(\varphi_{0}\right)=-F\left(\varphi_{0}+\pi\right)$.

If, again, the carrier repetition frequency is high compared to $\left|k V_{\mathrm{i}}\right|_{\max }$, the low frequency output from the mixer $G(\varphi)$ can be calculated as the mean value of the product $V_{1} F\left(\varphi_{0}\right), \varphi$ denoting the phase 
difference between the VCO and carrier. Neglecting amplitude factors we get for $G(\varphi)$

$$
\begin{aligned}
G(\varphi) & =\frac{1}{2 \pi}\left[\int_{\varphi}^{\varphi+\pi} F\left(\varphi_{0}\right) \mathrm{d} \varphi_{0}-\int_{\varphi+\pi}^{\varphi+2 \pi} F\left(\varphi_{0}\right) \mathrm{d} \varphi_{0}\right] \\
\frac{\mathrm{d} G(\varphi)}{\mathrm{d} \varphi} & =-\frac{2}{\pi} F(\varphi) .
\end{aligned}
$$

The functional dependence now appearing in eq. (1) can thus be determined by integrating the carrier waveform.

2. Lock range. - For an applied DC current $J_{i}<J_{1}$ the loop will act as a servo system adjusting the phase of the VCO relative to the carrier such that $J_{i}=J_{1} \sin \varphi$. The lock range $J_{1}$ corresponds to the supercurrent in a Josephson junction. The loop gain $A(\omega)$ can be calculated, breaking the loop at point $\mathrm{P}$ (Fig. 1). A small rf current $J_{\text {rf }}$ at angular frequency $\omega$ is applied to the input. For small phase deviations eq. (1) may be written

$$
\begin{aligned}
J & =J_{1} \sin \left(\varphi+\int k V_{\mathrm{i}} \mathrm{d} t\right) \\
& \simeq J_{1} \sin \varphi+J_{1} \cos \varphi \cdot \int k V_{\mathrm{i}} \mathrm{d} t,
\end{aligned}
$$

$\varphi$ denoting the mean phase. To calculate $A(\omega)$ the ac part is taken

$$
J_{\mathrm{ac}}=J_{1} \cos \varphi \cdot \int k V_{\mathrm{i}} \mathrm{d} t
$$

which in the usual $j \omega$-formalism is

$$
J_{\mathrm{ac}}=J_{1} \cos \varphi \cdot \frac{k R J_{\mathrm{rf}}}{j \omega},
$$

where $V_{\mathrm{i}}=R J_{\mathrm{rf}}$ has been inserted. Introducing $\omega_{1}=k R J_{1}$ loop gain becomes

$$
A(\omega)=\frac{J_{\mathrm{ac}}}{J_{\mathrm{rf}}}=\cos \varphi \cdot \frac{\omega_{1}}{j \omega} .
$$

The Nyquist diagram [2] of $A(\omega)$ is the positive or negative part of the imaginary axis, the latter corresponding to stable states ( $\cos \varphi$ positive).

The closed loop input admittance is $Y=Y^{\prime}(1+A)$, where $Y^{\prime}=1 / R$ is open loop input admittance

$$
Y=\frac{1}{R}+R^{-1} \cos \varphi \cdot \frac{\omega_{1}}{j \omega},
$$

i. e. equivalent to a resistance $R$ shunted by an inductance $L=R / \omega_{1} \cos \varphi=1 / k J_{1} \cos \varphi$.

A resonant circuit (the plasma resonance) is formed by connecting a capacitor $C$ across the input terminals. The plasma frequency $\omega_{\mathrm{p}}$ is given by

$$
\omega_{\mathrm{p}}=(L C)^{-1 / 2}=\left(\frac{k J_{1}}{C}\right)^{1 / 2}\left(1-\left(\frac{J_{\mathrm{DC}}}{J_{1}}\right)^{2}\right)^{1 / 4} .
$$

The bandwidth of the plasma resonance is in this model

$$
\frac{\omega_{\mathrm{p}}}{Q}=\frac{1}{R C}
$$

independent of $\cos \varphi$.

The plasma resonance may as well be considered as the natural frequency of oscillation in a second order loop.

3. I-V characteristic. - If the lock range $J_{1}$ is exceeded a DC voltage will develop across the input. Due to the feed-back the input voltage will oscillate with repetition frequency $\omega_{\mathrm{J}}=k U_{\mathrm{DC}}$ between the limits $R\left(J_{\mathrm{i}} \pm J_{1}\right)$. The input voltage will spend a major part of the time at low voltages, since $\mathrm{d} \varphi / \mathrm{d} t$ here is the lowest. This asymmetry is responsible for the DC current drawn by the junction element at finite voltages.

As $J_{\mathrm{i}}$ is lowered again to $J_{1}$, the input voltage will reach zero, reestablishing phase lock. It is seen that capture range and lock range are equal for the loop shown in figure 1.

Analytical expressions for the waveforms and the $I-V$ characteristic are given in reference [4].

A sizeable capacitor across the input affects the $I-V$ characteristic in two ways, short-circuiting the ac feed-back :

i) The voltage switches from zero to $R J_{\mathbf{i}}$, if the lock range is exceeded, since the junction can no longer carry DC-current.

ii) Due to the missing feed-back, phase-lock is not reestablished until $J_{1}$ is decreased below $J_{1}$. For a second order loop the capture range differs from the lock range.

In the $I-V$ characteristic the effect appears as hysteresis [5], [6].

4. rf-induced steps. - In a sinusoidal rf voltage with amplitude $V_{\mathrm{rf}}$ and angular frequency $\omega_{\mathrm{rf}}$ is applied to the input, the VCO will be frequency modulated. According to standard FM-theory the following spectrum is produced :

center frequency $\omega_{0}$ with relative amplitude $J_{0}(m)$ sidebands $\omega_{0} \pm n \omega_{\mathrm{rf}}$ with relative amplitude $J_{n}(m)$

where the modulation index $m$ is given by

$$
m=\frac{\Delta \omega_{\mathrm{vco}}}{\omega_{\mathrm{rf}}}=\frac{k \cdot V_{\mathrm{rf}}}{\omega_{\mathrm{rf}}} .
$$

Phase locking may now occur not only for zero DC voltage but for any DC voltage shifting one of the sidebands to the carrier frequency $\omega_{0}$, i. e. for

$$
U_{\mathrm{DC}}= \pm \frac{n \omega_{\mathrm{rf}}}{k}
$$


the corresponding lock range being proportional to $J_{n}(m)$. It should be noted that RF-induced steps may be considered as shifted supercurrents. They do not differ in nature from the zero voltage supercurrent.

If the rf-drive is not a constant voltage drive, the pattern is more complicated [3] since the input voltage then will be modified by the feed-back. In the limit $\omega_{\mathrm{rf}} \gg \omega_{1}$ the voltage is only slightly affected, since the loop gain, as can be seen from eq. (3), is below unity. The RF induced steps show the regular Bessel function dependence on applied rf voltage. In the limit $\omega_{\text {rf }} \ll \omega_{1}$ the feed-back contributes significantly to the total input voltage. Consider a step at a DC voltage

$$
U_{\mathrm{DC}}=\frac{n \omega_{\mathrm{rf}}}{k}
$$

and the corresponding Josephson frequency

$$
\omega_{\mathrm{J}}=k \cdot U_{\mathrm{DC}}=n \cdot \omega_{\mathrm{rf}} .
$$

The feed-back signal at frequency $\omega_{\mathrm{J}}$ modulates the VCO, producing its first sideband at $\omega_{0}+n \omega_{\mathrm{rf}}$. This sideband adds to the n'th sideband from the initial rf modulation to modify the step height.

The condition of constant voltage drive mentioned previously is contained in the inequality

$$
\omega_{\mathrm{rf}} \gg \omega_{1}, \quad \omega_{1}=k R J_{1}
$$

if $R$ is the total resistance seen by the junction.

5. $\operatorname{Cos} \varphi$-term. - The low-pass filter in figure 1 is now taken into account. Using a first order filter with transfer function $\left(1+j \omega / \omega_{\mathrm{c}}\right)^{-1}$, the loop gain $A(\omega)$ becomes

$$
A(\omega)=\cos \varphi \cdot\left(1+\frac{j \omega}{\omega_{\mathrm{c}}}\right)^{-1} \frac{\omega_{1}}{j \omega} .
$$

The Nyquist diagram of $A(\omega)$ is shown in figure 2. The effect of the filter is to push the gain curve away from the imaginary axis thus introducing a negative conductance component in the input admittance, since

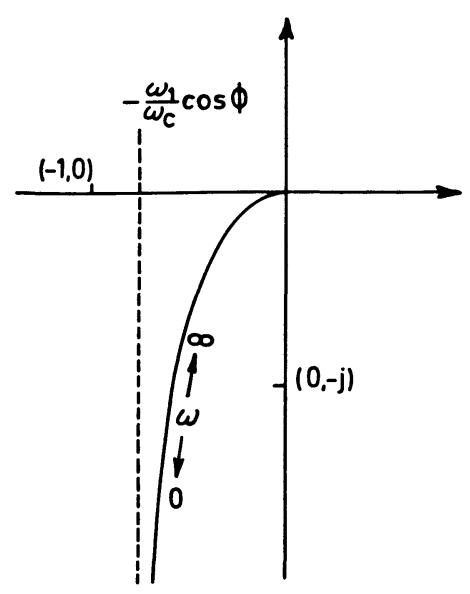

FIG. 2. - The Nyquist diagram. A plot of the loop gain $A(\omega)$ in the complex plane with $\omega$ as a parameter. again $Y=R^{-1}(1+A(\omega))$. Separating real and imaginary parts the following expression for $Y$ is obtained :

$$
\begin{aligned}
& Y=\frac{1}{j \omega} \frac{\omega_{1} \cos \varphi}{R\left[1+\left(\omega / \omega_{\mathrm{c}}\right)^{2}\right]}+ \\
& +\frac{1}{R}-\frac{1}{R} \frac{\omega_{1}}{\omega_{\mathrm{c}}} \frac{\cos \varphi}{\left[1+\left(\omega / \omega_{\mathrm{c}}\right)^{2}\right]}
\end{aligned}
$$

A discussion of the last term of eq. (4) has been given in reference [7].

Connecting a capacitor $\mathrm{C}$ across the input terminals the plasma frequency $\omega_{\mathrm{p}}$ is given by

$$
\omega_{\mathrm{p}}^{2}=\frac{\omega_{1}}{R C} \frac{\cos \varphi}{1+\left(\omega_{\mathrm{p}} / \omega_{\mathrm{c}}\right)^{2}}
$$

and the bandwidth of the plasma resonance

$$
\frac{\omega_{\mathrm{p}}}{Q}=\frac{1-\cos \varphi\left[1+\left(\omega_{\mathrm{p}} / \omega_{\mathrm{c}}\right)^{2}\right]^{-1} \omega_{1} / \omega_{\mathrm{c}}}{R C}
$$

For $\omega_{\mathrm{p}} \ll \omega_{\mathrm{c}}$ the bandwidth depends linearly on $\cos \varphi$ [8]. From (5) and (6) we get

$$
\frac{\omega_{\mathrm{p}}}{Q}=\frac{1}{R C}-\frac{\omega_{\mathrm{p}}^{2}}{\omega_{\mathrm{c}}}
$$

Eq. (7) may be used to determine the cut-off frequency $\omega_{\mathrm{c}}$ experimentally. The bandwidth $\omega_{\mathrm{p}} / Q$ and the plasma frequency $\omega_{\mathrm{p}}$ is measured for different values of $\cos \varphi$ (different values of DC-current) and $\omega_{\mathrm{p}} / Q$ is plotted against $\omega_{\mathrm{p}}^{2}$ (Fig. 3). From the slope of the line $\omega_{\mathrm{c}}$ is determined.

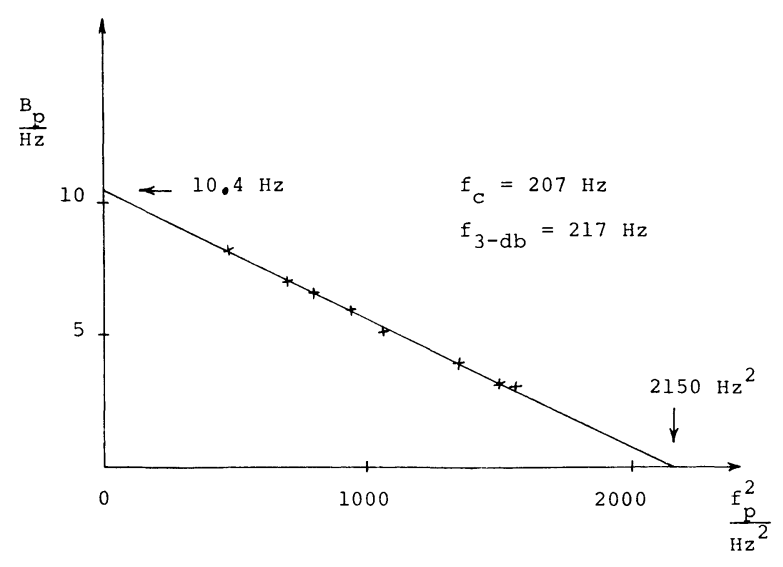

FIG. 3. - Bandwidth of the plasma resonance as measured on a junction analog.

The data presented in figure 3 were obtained using a low-frequency junction analog. For comparison the $3 \mathrm{~dB}$ cut-off frequency was measured by standard methods and found to agree with the result obtained using eq. (7).

Figure 4 represent data taken by N. F. Pedersen, T. F. Finnegan and D. N. Langenberg. The bandwidth $f_{\mathrm{p}} / Q$ of the plasma resonance is plotted versus $f_{\mathrm{p}}^{2}$. From the slope of the straight line a « junction cut-off 


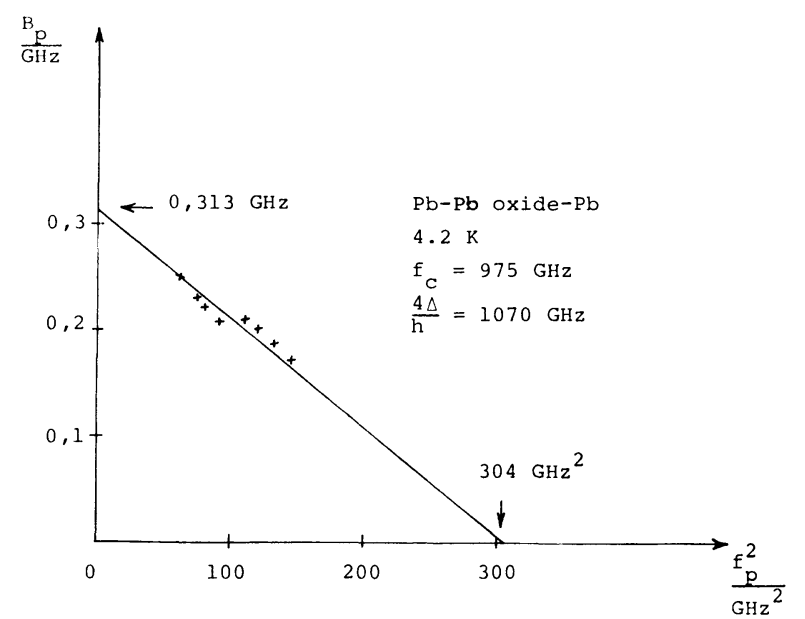

Fig. 4. - Bandwidth of the plasma resonance as measured on a lead-lead junction at $4.2 \mathrm{~K}$. frequency $" f_{\mathrm{c}}=975 \mathrm{GHz}$ is determined in fair agreement with the Riedel peak at $4 \Delta / h=1070 \mathrm{GHz}$.

6. Conclusion. - A fairly accurate equivalent circuit including an upper frequency limit has been presented. It is important to note that the junction can act as a current controlled inductance indicating the possibility of a replacement of the voltage controlled capacitance in parametric amplifiers.

A remaining problem in using Josephson junctions in microwave systems is, however, an efficient microwave coupling to the junction [9].

Acknowledgments. - I wish to thank Professor, Dr. Sidney Shapiro and Professor, Dr. K. Særmark for stimulating interest, Dr. N. F. Pedersen for supplying the data underlying figure 4 and L. Olsen for continuously updating electrical diagrams of various junction analogs.

\section{References}

[1] Auracher, F. and Van Duzer, T. Appl. Phys. Lett. 21 (1972) 515.

[2] Nyquist, H., Bell. Syst. Tech. J. 11 (1932) 126.

[3] Russer, P., J. Appl. Phys. 43 (1972) 2008.

[4] Solymar, L., Superconductive Tunneling and Applications (Chapman and Hall Ltd., London) 1972.

[5] Stewart, W. C., Appl. Phys. Lett. 12 (1968) 277.

[6] McCumber, D. E., J. Appl. Phys. 39 (1968) 3113.

[7] BaK, C. K. and Pedersen, N. F., Appl. Phys. Lett. 22 (1973) 149.

[8] Pedersen, N. F., Finnegan, T. F. and Langenderg, D. N., Proceedings of the XIII International Conference on Low Temperature Physics (ed. R. H. Kropschot and K. D. Timmerhaus), University of Colorado Press, Boulder (Colo.) (1972).

[9] Kofoed, B. and Sermark, K., to be published. 